\title{
Effect of pressure on the magnetic, transport, and thermal-transport properties of the electron-doped manganite $\mathrm{CaMn}_{1-x} \mathrm{Sb}_{x} \mathrm{O}_{3}$
}

\author{
Yuh Murano, ${ }^{1, \dagger}$ Michiaki Matsukawa, ${ }^{1, *}$ Syuya Ohuchi, ${ }^{1}$ Satoru Kobayashi, ${ }^{1}$ Shigeki Nimori, ${ }^{2}$ Ramanathan Suryanarayanan, ${ }^{3}$ \\ Keiichi Koyama, ${ }^{4}$ and Norio Kobayashi ${ }^{5}$ \\ ${ }^{1}$ Department of Materials Science and Engineering, Iwate University, Morioka 020-8551, Japan \\ ${ }^{2}$ National Institute for Materials Science, Tsukuba 305-0047, Japan \\ ${ }^{3}$ Laboratoire de Physico-Chimie de L'Etat Solide, CNRS,UMR8182 Universite Paris-Sud, F-91405 Orsay, France \\ ${ }^{4}$ Graduate School of Science and Engineering, Kagoshima University, Kagoshima 890-0065, Japan \\ ${ }^{5}$ Institute for Materials Research, Tohoku University, Sendai 980-8577, Japan
}

(Received 21 April 2010; revised manuscript received 27 January 2011; published 28 February 2011)

\begin{abstract}
We have demonstrated the effect of hydrostatic pressure on magnetic and transport properties, and thermaltransport properties in the electron-doped manganite $\mathrm{CaMn}_{1-x} \mathrm{Sb}_{x} \mathrm{O}_{3}$. The substitution of $\mathrm{Sb}^{5+}$ ion for $\mathrm{Mn}^{4+}$ site of the parent matrix causes one-electron doping with the chemical formula $\mathrm{CaMn}_{1-2 x}^{4+} \mathrm{Mn}_{x}^{3+} \mathrm{Sb}_{x}^{5+} \mathrm{O}_{3}$ accompanied by a monotonous increase in unit-cell volume as a function of $x$. Upon increasing the doping level of Sb, the magnitudes of both electrical resistivity and negative Seebeck coefficient are suppressed at high temperatures, indicating the electron doping. Anomalous diamagnetic behaviors at $x=0.05$ and 0.08 are clearly observed in the field cooled dc magnetization. The effect of hydrostatic pressure on dc magnetization is in contrast to the chemical pressure effect due to Sb doping. The dynamical effect of ac magnetic susceptibility measurement points to the formation of the magnetically frustrated clusters such as FM clusters embedded in canted AFM matrix.
\end{abstract}

DOI: 10.1103/PhysRevB.83.054437 PACS number(s): 75.50.-y, 75.47.Gk, 62.50.-p, 72.20.Pa

\section{INTRODUCTION}

Manganese oxides with perovskite structure have been extensively investigated since the discovery of the colossal magnetoresistance (CMR) effect. ${ }^{1}$ The spontaneous insulator to metal transition and its associated CMR effect are well explained on the basis of the double exchange (DE) model between $\mathrm{Mn}^{3+}$ and $\mathrm{Mn}^{4+}$ ions. ${ }^{2}$ Furthermore, the phase separation model, where the ferromagnetic (FM) metallic and antiferromagnetic (AFM) insulating clusters of competing electronic phases coexist, strongly supports experimental studies of manganites. ${ }^{3}$ The dynamic Jahn-Teller (JT) effect of $\mathrm{Mn}^{3+}$ ions plays a crucial role in the physics of manganites. ${ }^{4}$

$\mathrm{CaMnO}_{3}$, the end member of the $\mathrm{Ca}_{1-x} \mathrm{La}_{x} \mathrm{MnO}_{3}$ system, undergoes a G-type antiferromagnetic transition around $T_{N} \sim$ $120 \mathrm{~K}$ accompanied by a weak ferromagnetic component, ${ }^{5}$ where each spin of $\mathrm{Mn}$ ions is antiparallel to its nearest neighbors of Mn. In recent years, the electron-doped manganite system $(x<0.5)^{6}$ has attracted much attention because of the possibility of the magnetoresistance effect. Neutron measurements on $\mathrm{Ca}_{1-x} \mathrm{La}_{x} \mathrm{MnO}_{3}{ }^{7}$ have revealed the formation of the nanometric-scale FM clusters isolated within a G-type AFM matrix for sufficiently low doping $(x<0.03)$. Furthermore, for intermediate doping $(0.03 \leqslant x \leqslant 0.14)$, a canting of the G-type AFM structure occurs with the nanometric FM clusters. In addition to a large number of researches on the A-site substituted electron doped manganites, it is shown that substituting the $\mathrm{Mn}$ site of $\mathrm{CaMnO}_{3}$ with valence ions higher than $4+$ such as $\mathrm{CaMn}_{1-2 x}^{4+} \mathrm{Mn}_{x}^{3+} M_{x}^{5+} \mathrm{O}_{3}$, with $M=\mathrm{Nb}$, $\mathrm{Ta}, \mathrm{V}, \mathrm{Ru}^{8}{ }^{8-13}$ and $\mathrm{CaMn}_{1-3 x}^{4+} \mathrm{Mn}_{2 x}^{3+} M_{x}^{6+} \mathrm{O}_{3}$, with $M=\mathrm{Mo}$, $\mathrm{W}, 8,9,14-18$ creates $\mathrm{Mn}^{3+}$ ions, i.e., electrons leading to the CMR effect. The crystallographic and magnetic properties of the $\mathrm{CaMn}_{1-x} \mathrm{Ru}_{x} \mathrm{O}_{3}$ system $(x \geqslant 0.1)$ allow us to apply the phase separated FM + AFM model to the ground state of this system. ${ }^{10}$ For the $\mathrm{CaMn}_{1-x} \mathrm{Mo}_{x} \mathrm{O}_{3}$ system with low doping of Mo $(x=0.04)$, the low temperature magnetic ground state is better described by the canted AFM magnetic structure than by the phase separated state. ${ }^{17,18}$ At higher doping levels of Mo, the charge ordered state is established within the parent matrix $\mathrm{CaMnO}_{3} \cdot{ }^{14}$ It thus is interesting to examine the physical properties of the Mn-site substituted compositions for our understanding of electronic phase diagram of electron-doped manganites.

In this paper, we demonstrate the effect of hydrostatic pressure on magnetic and transport properties, and thermal-transport properties in electron-doped manganites $\mathrm{CaMn}_{1-x} \mathrm{Sb}_{x} \mathrm{O}_{3}$, in order to examine a relationship between lattice and spin. The physical pressure effect is a powerful probe to investigate the electronic states of manganese oxides varying the one-electron band width at fixed doping level because the application of external pressure gives rise to a shrinkage of Mn-O bond length and/or straightening of a Mn-O-Mn bond angle. ${ }^{19}$ There have been several studies on the effect of pressure on the magnetism of calcium-based electron-doped manganites so far. ${ }^{20}$ For $\mathrm{CaMn}_{1-x} \mathrm{Ru}_{x} \mathrm{O}_{3}$ with $x=0.1$, the applied pressure dramatically suppresses the ferromagnetic phase accompanied by a rise of the magnetic transition temperature up to $\sim 14 \mathrm{~K}$.

In previous works of slightly electron-doped $\mathrm{CaMnO}_{3}$ with B-site substitution, negative magnetization properties have been demonstrated. ${ }^{12,21}$ The negative magnetization phenomena in manganites have been originally reported in compounds with two sublattices of $\mathrm{Mn}$ ions and rare-earth ions $(\mathrm{Nd}, \mathrm{Gd}$, $\mathrm{Dy})$, such as $\mathrm{NdMnO}_{3},{ }^{22,23}(\mathrm{La}, \mathrm{Gd}) \mathrm{MnO}_{3},{ }^{24}$ (Nd,Ca) $\mathrm{MnO}_{3},{ }^{25}$ (Gd,Ca) $\mathrm{MnO}_{3},{ }^{26}$ and (Dy,Ca) $\mathrm{MnO}_{3} .{ }^{27,28}$ Some of these studies were discussed on the basis of ferrimagnetic scenario leading to negative magnetization below a compensation temperature, where $\mathrm{Mn}$ and some rare-earth sublattices are 
antiferrmagnetically coupled. Moreover, a phase separation model between the ferromagnetic clusters and the canted AFM matrix is proposed, in order to account for a possible origin of the negative magnetization in manganites. ${ }^{22,25}$ In addition to the B-site substituted manganite, the negative magnetization was observed in some manganites without magnetic rare-earth ions, such as $\mathrm{LaMnO}_{3}$ nano particles. ${ }^{29}$ Accordingly, the nature of the negative magnetization in manganites is one of the crucial issues to be unveiled, which may be close to a phase-segregated state.

Furthermore, we carry out the ac magnetic susceptibility measurements for $\mathrm{CaMn}_{1-x} \mathrm{Sb}_{x} \mathrm{O}_{3}$, in order to examine the dynamic effect linked to magnetically frustrated properties. For a spin-glass or cluster-glass system, a visible anomaly in the ac susceptibility appears upon lowering $T$ across freezing temperature of spins or clusters when the magnetic relaxation time becomes longer than the measuring time. ${ }^{30}$ In particular, we believe that a phase-separated state realized in manganites is not consistent with a typical spin-glass phase at low field. ${ }^{31}$ It thus is very intriguing to demonstrate a close relationship between static and dynamic responses of magnetization to the applied field, for our further understanding of complicated magnetic behavior of the present samples.

\section{EXPERIMENT}

Polycrystalline samples of $\mathrm{CaMn}_{1-x} \mathrm{Sb}_{x} \mathrm{O}_{3} \quad(x=0.02$, $0.05,0.08$, and 0.1 ) were prepared with a solid-state reaction method. The stoichiometric mixtures of $\mathrm{CaCO}_{3}, \mathrm{Mn}_{3} \mathrm{O}_{4}$, and $\mathrm{Sb}_{2} \mathrm{O}_{3}$ high-purity powders were calcined in air at $1000^{\circ} \mathrm{C}$ for $24 \mathrm{~h}$. The products were then ground and pressed into cylindrical pellets. The pellets were finally sintered at $1400 \sim$ $1450{ }^{\circ} \mathrm{C}$ for $12 \mathrm{~h}$. X-ray diffraction data revealed that all samples are almost single phase with orthorhombic structures (Pnma) (Fig. 1). The lattice parameters of the $x=0.08$ sample are $a=5.326 \AA, b=7.512 \AA$, and $c=5.310 \AA$, which is in fair agreement with a previous work. ${ }^{32}$ The cell parameters and unit-cell volume increase with increasing $\mathrm{Sb}$ doping because the ion radius of $\mathrm{Sb}^{5+}(0.61 \AA)$ is greater than the value of $\mathrm{Mn}^{4+}(0.54 \AA)$. In addition, the $\mathrm{Mn}^{4+}$ ions are replaced by $\mathrm{Mn}^{3+}$ ions $(0.645 \AA)$ with one extra electron, which contributes to the increased cell volume. The electrical resistivity was measured with a four-probe method. The Seebeck coefficient was determined from both measurements of a thermoelectric voltage and temperature difference along the longitudinal direction of the measured sample. The thermal conductivity was collected with a conventional heat-flow method. The $\mathrm{dc}$ and ac magnetization measurement was carried out using the commercial superconducting quantum interference device (SQUID) magnetometers both at Iwate University and National Institute for Materials Science. The ac magnetic susceptibility measurement for $x=0.02,0.05$, and 0.08 samples was measured as a function of frequency and dc magnetic field at the ac magnetic field of $0.5 \mathrm{mT}$. In particular, to remove the influence of the remanent magnetic field, the SQUID magnetometer with the option of a magnet reset mode was used in low-field measurements. ${ }^{21,33}$ Hydrostatic pressures in magnetization and electrical resistivity measurements were applied by using a clamp-type $\mathrm{CuBe}$ cell up to $1 \mathrm{GPa}$. Fluorinert was used as a pressure transmitting medium.
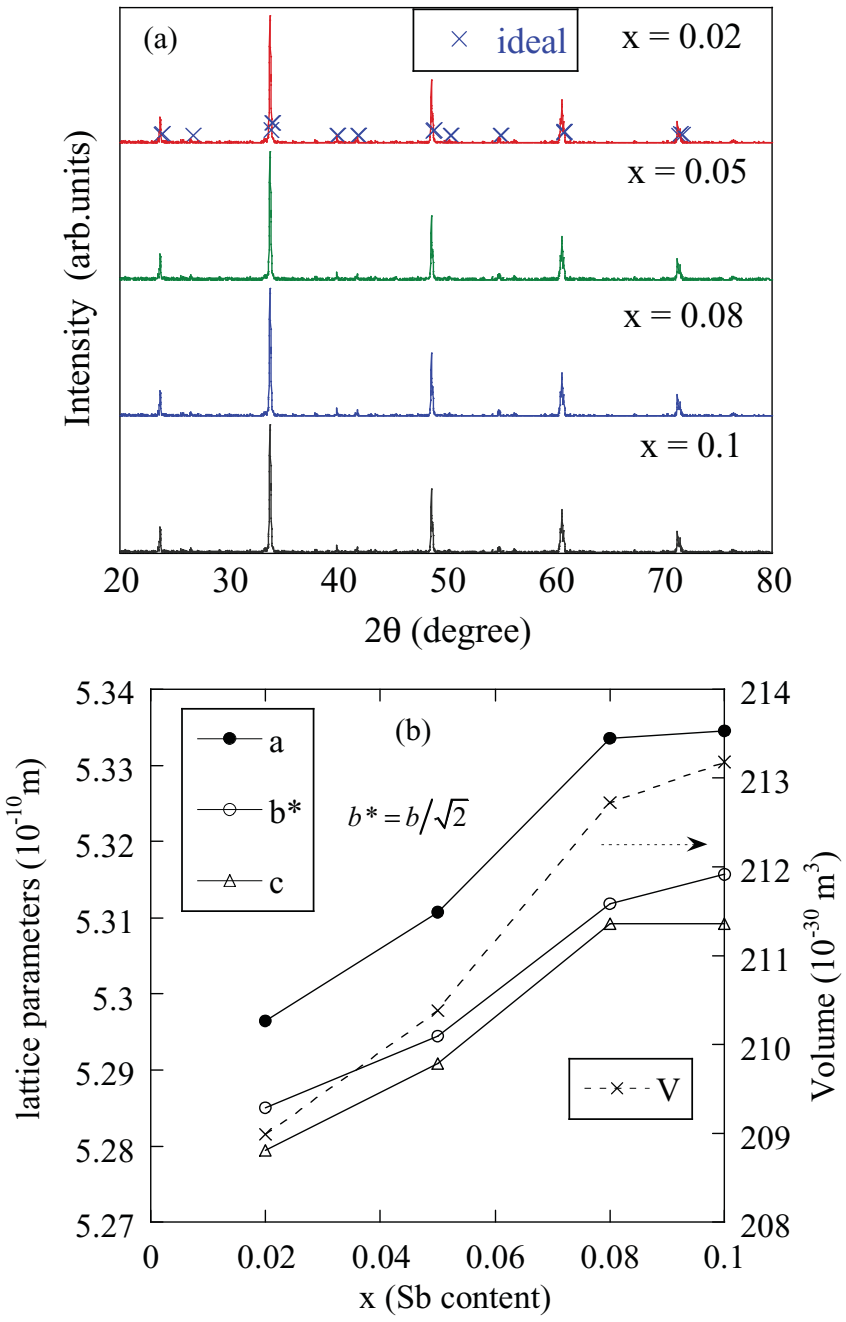

FIG. 1. (Color online) (a) X-ray diffraction patterns of $\mathrm{CaMn}_{1-x} \mathrm{Sb}_{x} \mathrm{O}_{3}(x=0.02,0.05,0.08$, and 0.1$)$ taken at room temperature. Cross marks denote intensity peaks of orthorhombic $\mathrm{CaMnO}_{3}$. (b) The lattice parameters and unit-cell volume as a function of $\mathrm{Sb}$ contents from 0.02 up to 0.1 .

The magnitude of pressure was calibrated by the pressure dependence of the critical temperature of lead. The magnetoresistance effect was measured by using a superconducting magnet at the High Field Laboratory for Superconducting Materials, Institute for Materials Research, Tohoku University.

\section{RESULTS AND DISCUSSION}

\section{A. Effect of magnetic field and external pressure on electrical transport}

The temperature variation of electrical resistivity $\rho$ for the $\mathrm{CaMn}_{1-x} \mathrm{Sb}_{x} \mathrm{O}_{3}$ system is shown in Fig. 2 as a function of the $\mathrm{Sb}$ content. The $\mathrm{Sb}$ substitution for the $\mathrm{Mn}$ site up to $x=0.1$ gives rise to a substantial decrease in high temperature $\rho(300 \mathrm{~K})$ from $1.5 \Omega \mathrm{cm}$ at the parent $x=0$ sample through $0.057 \Omega \mathrm{cm}$ at the lightly doped $x=0.02$ sample down to $0.044 \Omega \mathrm{cm}$ at the intermediately doped $x=0.1$ sample, indicating the carrier doping into the $\mathrm{Mn}$ site. All samples exhibit semiconducting behaviors upon decreasing temperatures except for a metallic conduction at 

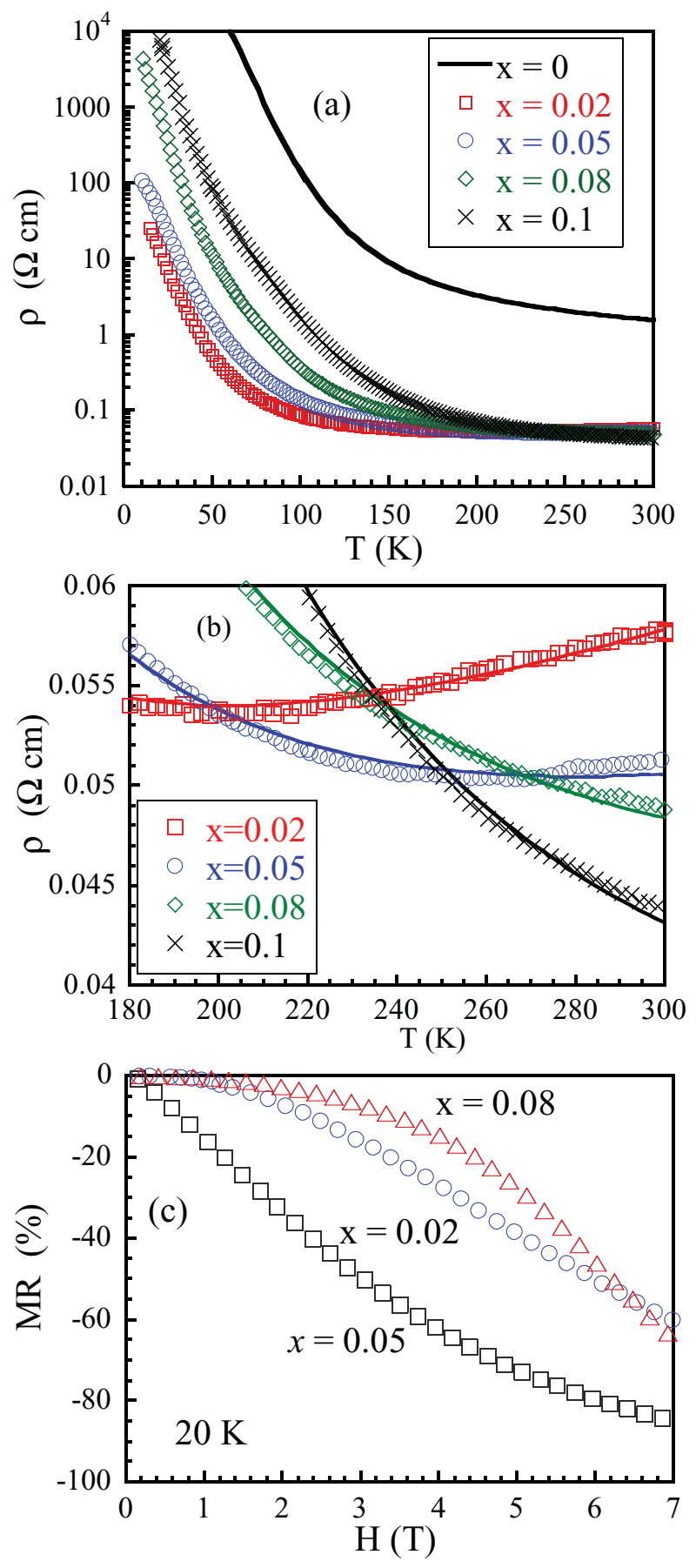

FIG. 2. (Color online) (a) Temperature variation of electrical resistivity $\rho$ for the $\mathrm{CaMn}_{1-x} \mathrm{Sb}_{x} \mathrm{O}_{3}$ system $(x=0.02,0.05,0.08$, and 0.1 . For comparison, the data of the parent compound are presented. (b) The $\rho-T$ plot is magnified between $180 \mathrm{~K}$ and room temperature. The solid lines denote the fits of the high- $T$ data by using a small polaron model. The activation energy $W$ is listed as a function of the $\mathrm{Sb}$ content in Table I. (c) Magnetoresistance effect, $M R=[R(H)-R(0)] / R(0) \times 100 \%$ at $20 \mathrm{~K}$ for the $\mathrm{CaMn}_{1-x} \mathrm{Sb}_{x} \mathrm{O}_{3}$ system $(x=0.02,0.05$, and 0.08$)$.

high temperatures for the $\mathrm{Sb}$ light doping. The $\rho-T$ curve of the $x=0.02$ and 0.05 samples remains a metallic-like character in the paramagnetic region down to $\sim 200 \mathrm{~K}$ and $250 \mathrm{~K}$, respectively. On the other hand, the magnitude of
TABLE I. Electrical transport characteristics of the $\mathrm{CaMn}_{1-x} \mathrm{Sb}_{x} \mathrm{O}_{3}$ series. $\rho_{300 \mathrm{~K}}$ represents the resistivity at $300 \mathrm{~K} . W$ is calculated from the best fits of the high- $T$ transport data using the small polaron model. The $M R$ under an applied field of $7 \mathrm{~T}$ and $\Delta R / R$ under an applied pressure of $0.8 \mathrm{GPa}$ are estimated from the effect of magnetic field and pressure on the resistivity, respectively. (For detail, see the captions of Figs. 2 and 3). Magnetic transition temperature $T_{m}$ is estimated from the inflection point of the field cooled $M-T$ curve of Fig. 5(a), 5(c), and 5(e).

\begin{tabular}{llcccr}
\hline \hline $\begin{array}{l}\text { Content } \\
x\end{array}$ & $\begin{array}{c}\rho_{300 \mathrm{~K}} \\
(\Omega \mathrm{cm})\end{array}$ & $\begin{array}{c}W \\
(\mathrm{meV})\end{array}$ & $\begin{array}{c}M R_{20 \mathrm{~K}} \\
(\%)\end{array}$ & $\begin{array}{c}\Delta R / R_{100 \mathrm{~K}} \\
(\%)\end{array}$ & $\begin{array}{c}T_{m} \\
(\mathrm{~K})\end{array}$ \\
\hline 0 & 1.5 & & & & 120 \\
0.02 & 0.057 & 17.4 & -60 & -17 & 110 \\
0.05 & 0.051 & 24.2 & -85 & -22 & 99 \\
0.08 & 0.049 & 33.9 & -64 & -47 & 88 \\
0.1 & 0.044 & 45.2 & & -43 & 38 \\
\hline \hline
\end{tabular}

the resistivity of $x=0.08$ and 0.1 is enhanced at lower temperatures since the further doping of $\mathrm{Sb}$ ion breaks some of conduction paths along the Mn-O-Mn network, resulting in a reinforcing carrier localization. In previous works, ${ }^{34,35}$ it is reported that the high-temperature transport in doped manganites is described by a small polaron hopping model. Now, we try to fit the resistivity data at high temperatures by using such an expression as $\rho=A T \exp (W / k T)$, where the fitting parameter $W$ represents the activation energy. As listed in Table I, with increasing Sb concentration, the value of the activation energy shows a gradual increase from $17.4 \mathrm{meV}$ at $x=0.02$ up to $45.2 \mathrm{meV}$ at $x=0.1$ in spite of a monotonous suppression in the resistivity vs Sb content at high temperatures. In the following section of thermal transport, we will discuss the transport mechanism in the $\mathrm{CaMn}_{1-x} \mathrm{Sb}_{x} \mathrm{O}_{3}$ system. We notice that the resistivity data of parent $\mathrm{CaMnO}_{3}$ observed here are, both in temperature dependence and magnitude, similar to the $\rho-T$ curve of the stoichiometric composition without oxygen defects, $\mathrm{CaMnO}_{3-\delta}(\delta=0) .{ }^{36}$

Next, let us examine the negative magnetoresistance effect on the electron-doped manganite samples with $x=0.02,0.05$, and 0.08 as shown in Fig. 2(c). At the $x=0.05$ sample, the gigantic magnetoresistance attaining $-85 \%$ at $20 \mathrm{~K}$ under the applied field of $7 \mathrm{~T}$ is observed. For the other samples, we obtain the MR ratio of $\sim-60 \%$. The MR data are comparable to those reported in a previous work ${ }^{8}$ on the $\mathrm{CaMn}_{1-x} M_{x} \mathrm{O}_{3}$ ( $M=\mathrm{Nb}$,Ta) system. These findings strongly indicate that the spin-polarized ferromagnetic metal clusters are established by the applied field and the field-induced delocalized state is realized within the samples studied.

Finally, shown in Fig. 3 is the effect of pressure on the electrical resistivity $\rho$ as a function of temperature between $77 \mathrm{~K}$ and $300 \mathrm{~K}$ for the $\mathrm{CaMn}_{1-x} \mathrm{Sb}_{x} \mathrm{O}_{3}(x=0.02$ and 0.08$)$. For comparison, the data at ambient pressure are presented. The applied pressure of $0.8 \mathrm{GPa}$ on the $x=0.08$ sample suppresses the magnitude of $\rho$ from $25 \%$ at $300 \mathrm{~K}$ down to about $50 \%$ at lower temperatures around $80 \mathrm{~K}$ upon decreasing temperature. The effect of pressure on the electrical transport observed here seems to be more enhanced below the magnetic transition temperatures $T_{m}=110 \mathrm{~K}$ at $x=0.02$ and $88 \mathrm{~K}$ at $x=0.08$, where $T_{m}$ is determined from the inflection point 

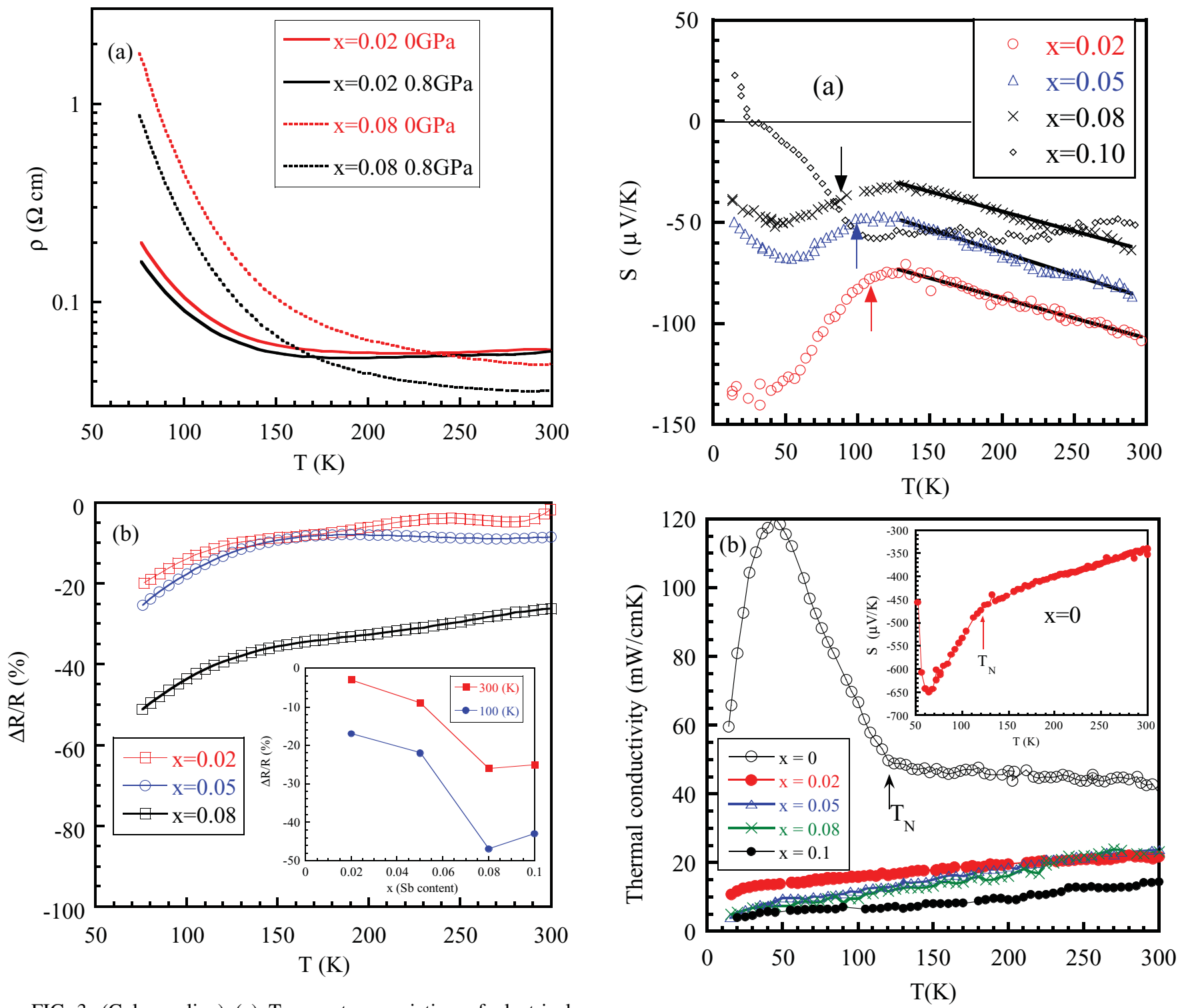

FIG. 3. (Color online) (a) Temperature variation of electrical resistivity $\rho$ for the $\mathrm{CaMn}_{1-x} \mathrm{Sb}_{x} \mathrm{O}_{3}$ system $(x=0.02$ and 0.08$)$ at an applied pressure of $0.8 \mathrm{GPa}$. For comparison, the data at ambient pressure are presented. (b) $\Delta R / R=[R(T, 0.8 \mathrm{GPa})-$ $R(T, 0 \mathrm{GPa})] / R(T, 0 \mathrm{GPa}) \times 100 \%$ for the $\mathrm{CaMn}_{1-x} \mathrm{Sb}_{x} \mathrm{O}_{3}$ system ( $x=0.02,0.05$, and 0.08$)$. The inset displays $\Delta R / R$ as a function of Sb content at $100 \mathrm{~K}$ and $300 \mathrm{~K}$.

of the field cooled magnetization curve under $10 \mathrm{mT}$. The pressure dependence of the resistance $\Delta R / R$ (the inset of Fig. 3) exhibits a maximum at the heavily doped sample of $x=0.08$, which is consistent with the effect of strong pressure on the magnitude of magnetization below $T_{m}$ [see Figs. 5(e) and 5(f)].

\section{B. Thermal transport (Seebeck coefficient and thermal conductivity)}

Seebeck coefficient $S$ is suppressed upon increasing $\mathrm{Sb}$ doping as shown in Fig. 4(a). For all samples studied, $S$ shows a negative value over a wide range of temperatures, strongly suggesting electron doping into the parent matrix. In the case of the substitution of $\mathrm{Sb}^{5+}$ ion for $\mathrm{Mn}^{4+}$ site, the

FIG. 4. (Color online) (a) Temperature variation of Seebeck coefficient $S$ for the $\mathrm{CaMn}_{1-x} \mathrm{Sb}_{x} \mathrm{O}_{3}$ system $(x=0.02,0.05,0.08$, and 0.1 ). The solid lines denote the $T$ linear fits to the $S(T)$ data in the temperature region above $130 \mathrm{~K}$. The arrows denote the magnetic transition temperatures determined from the magnetization data. (b) Thermal conductivity $\kappa$ of $\mathrm{CaMn}_{1-x} \mathrm{Sb}_{x} \mathrm{O}_{3}(x=0,0.02,0.05$, 0.08 , and 0.1 ). For comparison, the $\kappa$ data of the parent compound $\mathrm{CaMnO}_{3}$ are presented. The inset also represents the $S$ data of parent $\mathrm{CaMnO}_{3}$.

$\mathrm{Mn}^{4+}$ ions are replaced by $\mathrm{Mn}^{3+}$ ions with one extra electron. For lower doped samples $(x=0.02$ and 0.05$)$, the local maximum observed in $S$ is located near the magnetic transition temperature as mentioned above. With further doping of $\mathrm{Sb}$, the local maximum suppressed in $S$ of $x=0.08$ is still located around $130 \mathrm{~K}$, but it has little relationship with the weakly magnetic transition near $90 \mathrm{~K}$. We notice a common feature in the $T$-linear dependence of the Seebeck coefficient for three samples $(x=0.02,0.05$, and 0.08$)$ between $130 \mathrm{~K}$ and room temperature, although their $T$ dependences of the resistivity are quite different over the same temperature range as shown 
in Fig. 2(b). In our previous section, we demonstrated that the high- $T$ resistivity is well fitted by using a small polaron model. The corresponding expression for the Seebeck coefficient is given in the form of

$$
S(T)=\frac{k_{B}}{e}\left\{\frac{E_{S}}{k_{B} T}\right\}+S_{\infty},
$$

where $E_{S}$ and $S_{\infty}$ represent the thermal activation energy and the spin entropy in the high-temperature limit, respectively. ${ }^{34}$ In Fig. 4, we note that the $1 / T$ law is violated at high temperatures. The electrical resistivity of the present samples is greatly influenced by grain boundaries in comparison to the Seebeck measurement, giving rise to little reliable information about the electronic states. ${ }^{37}$ In fact, such disagreements between these transports of polycrystalline oxide materials have been pointed out thus far. ${ }^{38,39}$

In previous works ${ }^{35,38}$ of electron-doped manganites, the $T$-linear dependence of $S(T)$ has been discussed on the basis of the conventional metal model, or Culter and Mott (CM) model. The former is given in the form of

$$
S(T)=\pi^{2} \frac{k_{B}}{3 e}\left\{k_{B} T \frac{N(E)}{n}+\text { const. }\right\}_{E=E_{F}},
$$

where $N(E)$ is the density of states and $n$ is the carrier density. ${ }^{35}$ The latter formula for the CM model is expressed as

$$
S(T)=-\pi^{2} \frac{k_{B}}{3 e}\left\{k_{B} T \frac{\partial \ln \left(\mu_{0} N(E)\right)}{\partial E}-\frac{\partial W}{\partial E}\right\}_{E=E_{F}},
$$

where $\mu=\mu_{0} \exp \left(-W(E) / k_{B} T\right)$ is an activated mobility and $W(E)$ is an activation energy. ${ }^{40}$ The CM model for the random hopping system well describes a random distribution of localized states of electrons around the Fermi level. The typical fitting parameters $N(E) / n$ and $\left\{\partial \ln \left(\mu_{0} N\right) / \partial E\right\}^{-1}$, for the conventional and CM models are listed in Table II. The fitted result shows that the $N(E) / n$ ratio is almost independent of the nominal composition. The discrepancies in the $x=0.02$ and 0.05 samples between $N(E) / n$ and $n(\mathrm{Mn}) / n\left(\mathrm{Mn}^{3+}\right)$ is probably related to the fact that a single-band model is not valid in the case of the low-carrier concentration. The magnitudes of $\left\{\partial \ln \left(\mu_{0} N\right) / \partial E\right\}^{-1}$ are similar to those of the A-site substituted manganite $\mathrm{Ca}_{1-x} \mathrm{La}_{x} \mathrm{MnO}_{3}(x=0.017$ and 0.033). ${ }^{38}$ For the insulating $\mathrm{Li}_{1+x} \mathrm{Ti}_{2-x} \mathrm{O}_{4}$ sintered samples,

TABLE II. Thermal transport characteristics of the $\mathrm{CaMn}_{1-x} \mathrm{Sb}_{x} \mathrm{O}_{3}$ series. $S_{300 \mathrm{~K}}$ represents Seebeck coefficient at $300 \mathrm{~K} . N(E) / n$ is calculated from the best fits of the high- $T$ data using the conventional model. $n(\mathrm{Mn}) / n\left(\mathrm{Mn}^{3+}\right)$ is the ratio of the number of total $\mathrm{Mn}$ ions to that of the $\mathrm{Mn}^{3+}$ ion. We obtain from the inverse slopes of $S,\left\{\partial \ln \left(\mu_{0} N\right) / \partial E\right\}^{-1}$, by using the CM model.

\begin{tabular}{lcccc}
\hline \hline $\begin{array}{l}\text { Content } \\
x\end{array}$ & $\begin{array}{c}S_{300 \mathrm{~K}} \\
(\mu \mathrm{V} / \mathrm{K})\end{array}$ & $\begin{array}{c}\frac{N(E)}{n} \\
(1 / \mathrm{eV})\end{array}$ & $\frac{n(\mathrm{Mn})}{n\left(\mathrm{Mn}^{3+}\right)}$ & $\begin{array}{c}\left(\frac{\partial \ln \left[\mu_{0} N(E)\right]}{\partial E}\right)^{-1} \\
(\mathrm{eV})\end{array}$ \\
\hline 0 & -340 & & & \\
0.02 & -107 & 8.2 & 50 & 0.12 \\
0.05 & -84 & 9.3 & 20 & 0.11 \\
0.08 & -63 & 8 & 12.5 & 0.13 \\
0.1 & -51 & & & \\
\hline \hline
\end{tabular}

it has been reported that $S(T)$ is proportional to temperature and the $S(T)$ behavior is analyzed using the CM model. ${ }^{39}$ The Sb-substitution-induced lattice disorder influences the electronic states of the parent matrix, causing the validity of the CM model. Concerning the B-site substituted manganites, there is a common trend in the temperature dependence of $S(T)$ between $\mathrm{CaMn}_{1-x} \mathrm{~W}_{x} \mathrm{O}_{3}(x=0.02$ and 0.04$)$ and the present samples with $x=0.02,0.05$, and $0.08 .^{16}$

For $x=0.1$, the $S(T)$ data remain a nearly constant from room temperature down to near $100 \mathrm{~K}$, then increase rapidly and finally exhibit a positive value across the horizontal axis at lower temperatures. Such an unusual behavior in $S(T)$ has been reported both in A-site substituted system $\mathrm{Ca}_{1-x} \mathrm{Sm}_{x} \mathrm{MnO}_{3}$ $(x=0.2)^{41}$ and B-site system $\mathrm{CaMn}_{1-x} \mathrm{~W}_{x} \mathrm{O}_{3}(x \geqslant 0.07),{ }^{16}$ indicating a holelike character of charge carriers.

Finally, let us show in Fig. 4 the temperature variation of thermal conductivity in $\mathrm{CaMn}_{1-x} \mathrm{Sb}_{x} \mathrm{O}_{3}(x=0,0.02$, $0.05,0.08$, and 0.1 ). The thermal conduction for all samples studied here is carried by acoustic phonons because the electron contribution is negligible by an estimation from the electric resistivity data using the Wiedemann-Franz law. Upon decreasing temperature crossing the antiferromagnetic temperature $T_{N}$, a sharp increase in $\kappa$ of parent $\mathrm{CaMnO}_{3}$ is observed as reported in previous works. ${ }^{41,42}$ We believe that the thermal conductivity in the paramagnetic phase is strongly suppressed by phonon scattering due to nanoscale strains generated by spin correlations. ${ }^{42}$ This finding is thus explained by a rapid reduction of the phonon scattering when the AFM long-range order is established below $T_{N}$. The light doping of $\mathrm{Sb}^{5+}$ strongly suppresses the magnitude of thermal conductivity from $120 \mathrm{~mW} / \mathrm{cmK}(50 \mathrm{~K})$ at the pure $x=0$ sample down to $14 \mathrm{~mW} / \mathrm{cmK}$ at $x=0.02$ (Fig. 4). The $\mathrm{Sb}^{5+}$ doping removes the $\mathrm{Mn}^{4+}$ ion and instead produces the $\mathrm{Mn}^{3+}$ ion for Mn sites which is the Jahn-Teller active ion with one $e_{g}$ electron. It is believed that the local lattice distortion due to the JT effect causes phonon scattering, which is close to the depressed thermal conduction. ${ }^{43}$ In addition, the lattice deformation due to the $\mathrm{Sb}$ doping with its larger ion radius affects the neighboring $\mathrm{Mn}^{3+} \mathrm{O}_{6}$ octahedron, resulting in some variation of the orbital state of the $e_{g}$ electron through the local JT effect.

\section{Effect of pressure and magnetic field on dc magnetization}

Let us show in Figs. 5(a), 5(c), and 5(e) the zero-fieldcooled (ZFC) and field-cooled (FC) magnetization data of the $\mathrm{Sb}$ substituted $\mathrm{CaMn}_{1-x} \mathrm{Sb}_{x} \mathrm{O}_{3}$ measured under an applied magnetic field of $10 \mathrm{mT}$ at $0 \mathrm{GPa}$. First of all, temperaturedependent magnetization reversal appears in the FC curves of both $x=0.05$ and 0.08 , as it has been reported in the V-doped CaMn ${ }_{1-x} \mathrm{~V}_{x} \mathrm{O}_{3}$ manganites. ${ }^{12}$ However, lowering the temperature down to $2 \mathrm{~K}$, the $\mathrm{ZFC}$ data of both samples show no negative value. Upon increasing the $\mathrm{Sb}$ content up to $x=0.1$, a large difference between $\mathrm{ZFC}$ and FC curves is observed but such negative magnetization phenomenon vanishes. The magnetic transition temperature $T_{m}$ is suppressed from $110 \mathrm{~K}$ at $x=0.02$, through $99 \mathrm{~K}$ at $x=0.05$, down to $38 \mathrm{~K}$ at $x=0.1$ due to the $\mathrm{Sb}$ doping because the $\mathrm{Sb}^{5+}$ ion is a nonmagnetic one with the closed shell of $4 d^{10}$. The substitution of nonmagnetic $\mathrm{Sb}$ ions for $\mathrm{Mn}$ sites introduces electron 


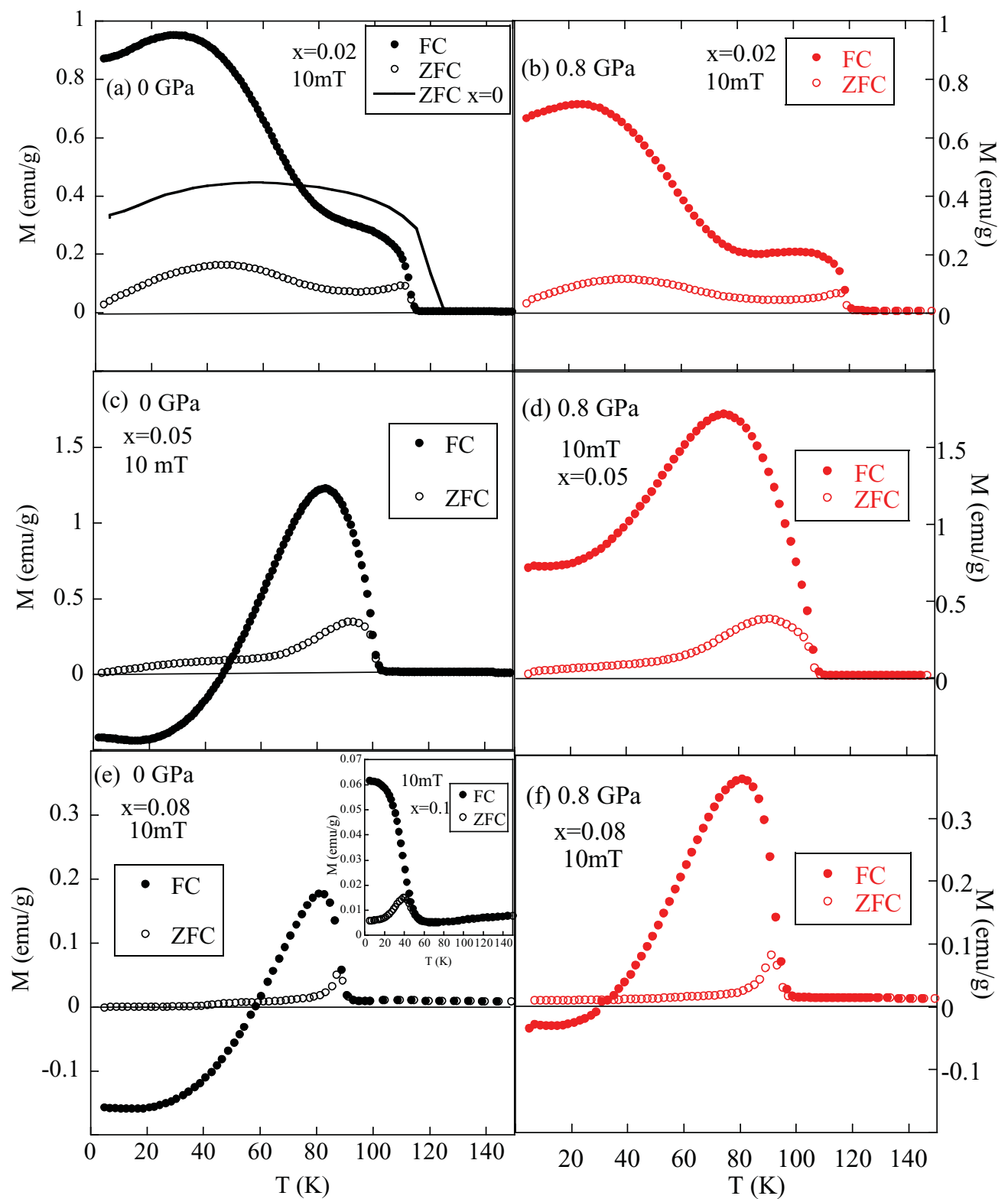

FIG. 5. (Color online) Temperature dependence of zero-field-cooled and field-cooled magnetization of the $\mathrm{Sb}$ substituted $\mathrm{CaMn}_{1-x} \mathrm{Sb}_{x} \mathrm{O}_{3}$ recorded at $H=10 \mathrm{mT}$. In the case of ambient pressure, (a) $x=0.02$, (c) $x=0.05$, and (e) $x=0.08$. In the case of $0.8 \mathrm{GPa}$, (b) $x=0.02$, (d) $x=0.05$, and (f) $x=0.08$. For comparison, the ZFC data of the parent sample are given in (a). In the inset of (e), the $M-T$ curve with the $x=0.1$ sample is presented at $0 \mathrm{GPa}$.

doping, forming ferromagnetic clusters, but destroys magnetic ordering between $\mathrm{Mn}$ ions within the parent matrix.

The FC data of $x=0.08$ indicates that the antiparallel component of the canted AFM spins to the applied field is stabilized by the $\mathrm{Sb}$ substitution. The spontaneously canted magnetic moment contributing to the negative magnetization is close to a variation of the local easy axis which is caused by the local lattice distortion of Sb substitution in comparison to the $\mathrm{Sb}$ free sample. If the number of canted spin clusters contributing to the antiparallel component is dominant over that of these clusters contributing to the parallel one along the applied field, we then obtain the negative magnetization. Furthermore, we need to point out the significant roles of the Dzyaloshinsky-Moriya interaction which prefers canted spin arrangements. If the antisymmetric exchange interaction between nearest-neighbor $\mathrm{Mn}$ ions is dominant, such mechanism then stabilizes noncollinear spin configuration, leading to complicated magnetic behaviors. In order to account for the negative magnetization observed in the lightly doped $\mathrm{Nd}_{1-x} \mathrm{Ca}_{x} \mathrm{MnO}_{3}$ series, the low-temperature spin reorientation is explained in the framework of a two-phase model, where the samples consist of ferromagnetic- and weakferromagnetic-phases exchange coupled at their boundaries. ${ }^{25}$ The reorientation of the magnetic moment of $\mathrm{Nd}$ ions in both phases plays a crucial role on the magnetization reversal through the $f-d$ exchange interaction between the $\mathrm{Nd}$ and 


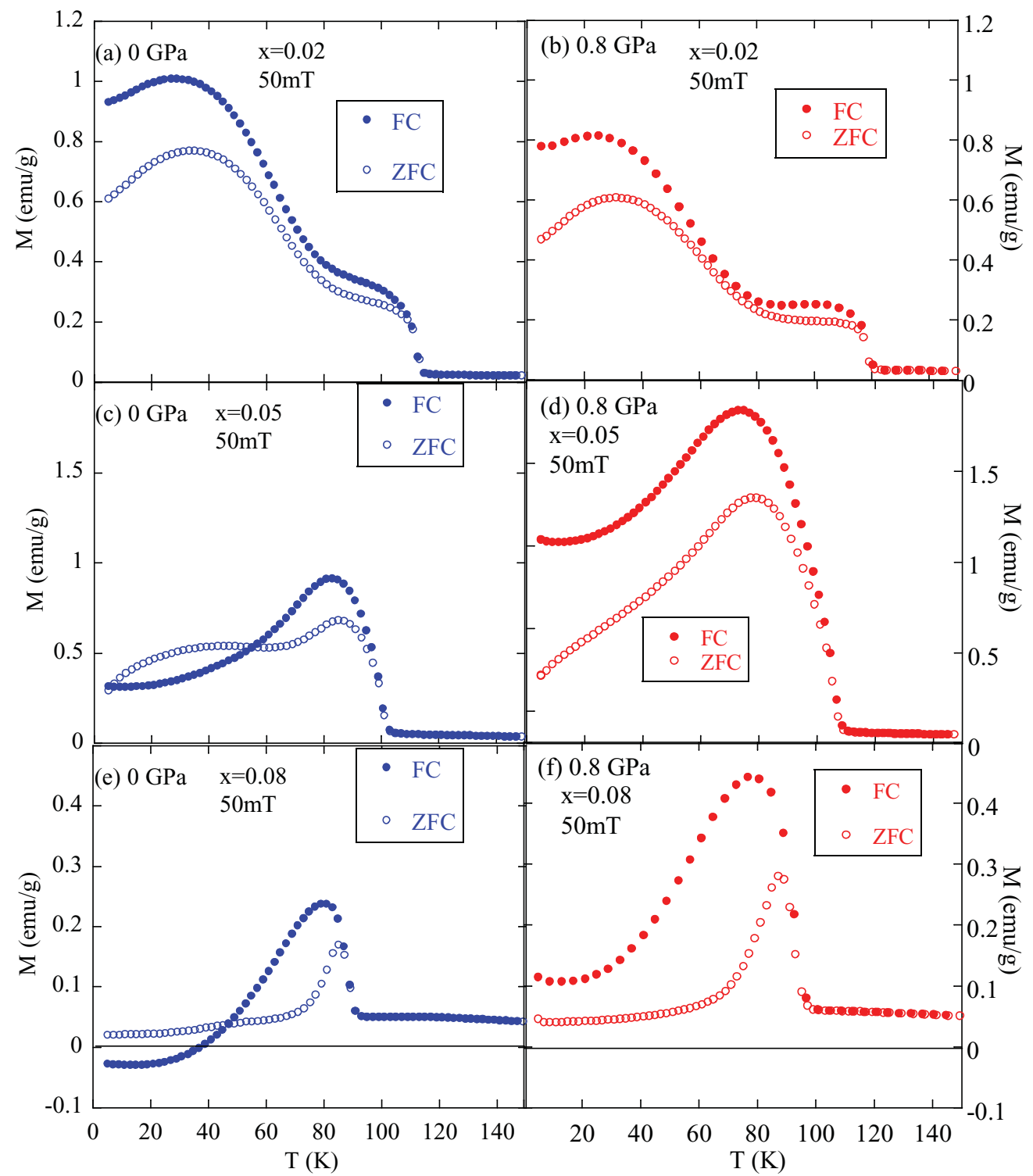

FIG. 6. (Color online) ZFC and FC magnetization curves of the Sb substituted $\mathrm{CaMn}_{1-x} \mathrm{Sb}_{x} \mathrm{O}_{3}(x=0.02,0.05$, and 0.08$)$ collected at $H=50 \mathrm{mT}$. For ambient pressure, we get (a) $x=0.02$, (c) $x=0.05$, and (e) $x=0.08$. For applied pressure of $0.8 \mathrm{GPa}$, (b) $x=0.02$, (d) $x=0.05$, and (f) $x=0.08$.

manganese sublattices. It seems that the proposal model is ruled out because the magnetic rare-earth ion is absent in the present system.

Next, we attempt to measure the effect of pressure on the magnetization for $\mathrm{CaMn}_{1-x} \mathrm{Sb}_{x} \mathrm{O}_{3}$, to examine a relation between lattice distortion and spin arrangement [Figs. 5(b), 5(d), and 5(f)]. The application of hydrostatic pressure up to $0.8 \mathrm{GPa}$ enhances $T_{m}$ by $8 \sim 9 \mathrm{~K}$ at $x=0.02$ and 0.05 , and a stable increase in $T_{m}$ reaches about $5 \mathrm{~K}$ at the $x=0.08$ sample. We expect that a shrinkage of lattice parameters makes the superexchange interaction stronger between nearest-neighbor $t_{2 g}$ spins of $\mathrm{Mn}^{4+}$ ions, resulting in a stabilization of the G-type AFM magnetic structure. The magnitude of magnetization is not largely changed at both $x=0.02$ and 0.05 . On the other hand, the maximum value of the FC curve of the $x=0.08$ sample is increased from $0.18 \mathrm{emu} / \mathrm{g}$ at $0 \mathrm{GPa}$ up to
$0.36 \mathrm{emu} / \mathrm{g}$ at $0.8 \mathrm{GPa}$, which is responsible for a sharp increase of FM clusters. Both the ZFC and FC curves at $x=0.02$ under $0.8 \mathrm{GPa}$ are, in temperature dependence, similar to those under ambient pressure, but the magnitude of the magnetization is suppressed by the application of pressure. The application of external pressure on the magnetization reversal of the $x=0.05$ sample causes the positive FC curve, approaching the ambient magnetization curve of the lowdoped sample $(x=0.02)$. At further Sb content of $x=0.08$, the negative magnetization of the FC curve at $0.8 \mathrm{GPa}$ is limited in the lower temperature region below $\sim 30 \mathrm{~K}$. However, it seems that the ZFC curves of all samples remain qualitatively unchanged even under the applied pressure. The orthorhombic distortion due to $\mathrm{Sb}$ doping is suppressed by an application of pressure, resulting in a similar magnetic behavior to the lower doped case under ambient pressure. At $H=50 \mathrm{mT}$, the 
negative magnetization disappears except for the lowtemperature $M T$ curve of $x=0.08$ at ambient pressure as shown in Fig. 6.

Here, we make remarks on a notable difference between the magnetic properties of the $x=0.1$ sample and other ones $(x=0.02,0.05$, and 0.08). As mentioned above, the magnetic transition temperature $T_{m}$ is monotonously decreased from $110 \mathrm{~K}$ at $x=0.02$ through $99 \mathrm{~K}$ at $x=0.05$ to $88 \mathrm{~K}$ at $x=0.08$ upon further $\mathrm{Sb}$ doping. At a maximum content of $x=0.1, T_{m}$ is strongly depressed down to $40 \mathrm{~K}$. These findings indicate that the magnetic interaction working between manganese ions is considerably suppressed beyond $x=0.08$. For the $x=0.1$ sample, the nominal content of the $e_{g}$ electron is taken as a maximum in all samples studied. It is true that the room-temperature resistivity and corresponding Seebeck coefficient of the $x=0.1$ sample exhibit their lowest values. However, upon lowering temperatures, the suppression of the magnetic coupling working among the $\mathrm{Mn}$ ions around each $\mathrm{Sb}$ ion probably becomes dominant over the carrier doping effect and causes a strongly reduced magnetization accompanied by a remarkable decrease of $T_{m}$. In addition to it, we point out the giant pressure effect on the magnitude of magnetization of the $x=0.08$ sample in applied fields of $10 \mathrm{mT}$ and $50 \mathrm{mT}$ as depicted in Figs. 5(d) and 6(d). In particular, the weakened magnetic interaction due to $\mathrm{Sb}$ doping under ambient pressure is strengthened under an applied pressure of $0.8 \mathrm{GPa}$, giving the remarkable increase in the magnetization by a factor of about 2 . The critical content is located near $x=0.08$, separating the negative and normal magnetic tendencies.

Let us determine from the $1 / M-T$ data taken at $H=$ $0.1 \mathrm{~T}$ (not shown here), the Curie-Weiss temperature and the effective magnetic moment, for the $\mathrm{CaMn}_{1-x} \mathrm{Sb}_{x} \mathrm{O}_{3}$ system $(x=0.02,0.05$, and 0.08) as listed in Table III. The CurieWeiss temperature $\Theta$ is estimated from a high-temperature linear fit of $1 / M-T$ by using the relation $\chi=C /(T-\Theta)$. The Curie constant $C$ gives the effective magnetic moment $\mu_{\text {exp }}$ per one manganese ion. For comparison, the effective magnetic moment $\mu_{\text {cal }}$ is calculated as a function of nominal Sb content by using $\mu_{\text {eff }}^{2}=(1-2 x) \mu^{2}\left(\mathrm{Mn}^{4+}\right)+x \mu^{2}\left(\mathrm{Mn}^{3+}\right)$. For free ions without the magnetic interaction, in the high-spin

TABLE III. Effect of pressure on magnetic transition temperature $T_{m}$ of the $\mathrm{CaMn}_{1-x} \mathrm{Sb}_{x} \mathrm{O}_{3}$ system $(x=0.02,0.05$, and 0.08$) . T_{m}$ and $T_{m}^{p}$ are determined from the inflection of $M-T$ curves of Fig. 5 under $0 \mathrm{GPa}$ and $0.8 \mathrm{GPa}$, respectively. $M_{\max }$ and $M_{\max }^{p}$ denote a maximum in FC magnetization curves below the magnetic transition temperatures under $0 \mathrm{GPa}$ and $0.8 \mathrm{GPa}$, respectively. $\Theta$ and $\mu_{\exp }$ represent the Curie-Weiss temperature and the effective magnetic moment per one manganese ion estimated from a high-temperature linear fit of $1 / M-T$ under ambient pressure. For comparison, the effective magnetic moment $\mu_{\text {cal }}$ is given (for detail, see the text).

\begin{tabular}{|c|c|c|c|c|c|c|c|}
\hline$x$ & $\begin{array}{c}T_{m} \\
(\mathrm{~K})\end{array}$ & $\begin{array}{l}T_{m}^{p} \\
(\mathrm{~K})\end{array}$ & $\begin{array}{c}M_{\max } \\
(\mathrm{emu} / \mathrm{g})\end{array}$ & $\begin{array}{c}M_{\max }^{p} \\
(\mathrm{emu} / \mathrm{g})\end{array}$ & $\begin{array}{c}\Theta \\
(\mathrm{K})\end{array}$ & $\begin{array}{c}\mu_{\exp } \\
\left(\mu_{B} / \mathrm{Mn}\right)\end{array}$ & $\begin{array}{c}\mu_{\mathrm{cal}} \\
\left(\mu_{B} / \mathrm{Mn}\right)\end{array}$ \\
\hline 0 & 120 & & & & & & 3.87 \\
\hline 0.02 & 110 & 119 & 0.95 & 0.71 & -119 & 3.51 & 3.85 \\
\hline 0.05 & 99 & 107 & 1.23 & 1.70 & -1 & 3.41 & 3.83 \\
\hline 0.08 & 88 & 93 & 0.18 & 0.36 & 28 & 3.70 & 3.81 \\
\hline 0.1 & 38 & & & & & & 3.80 \\
\hline
\end{tabular}
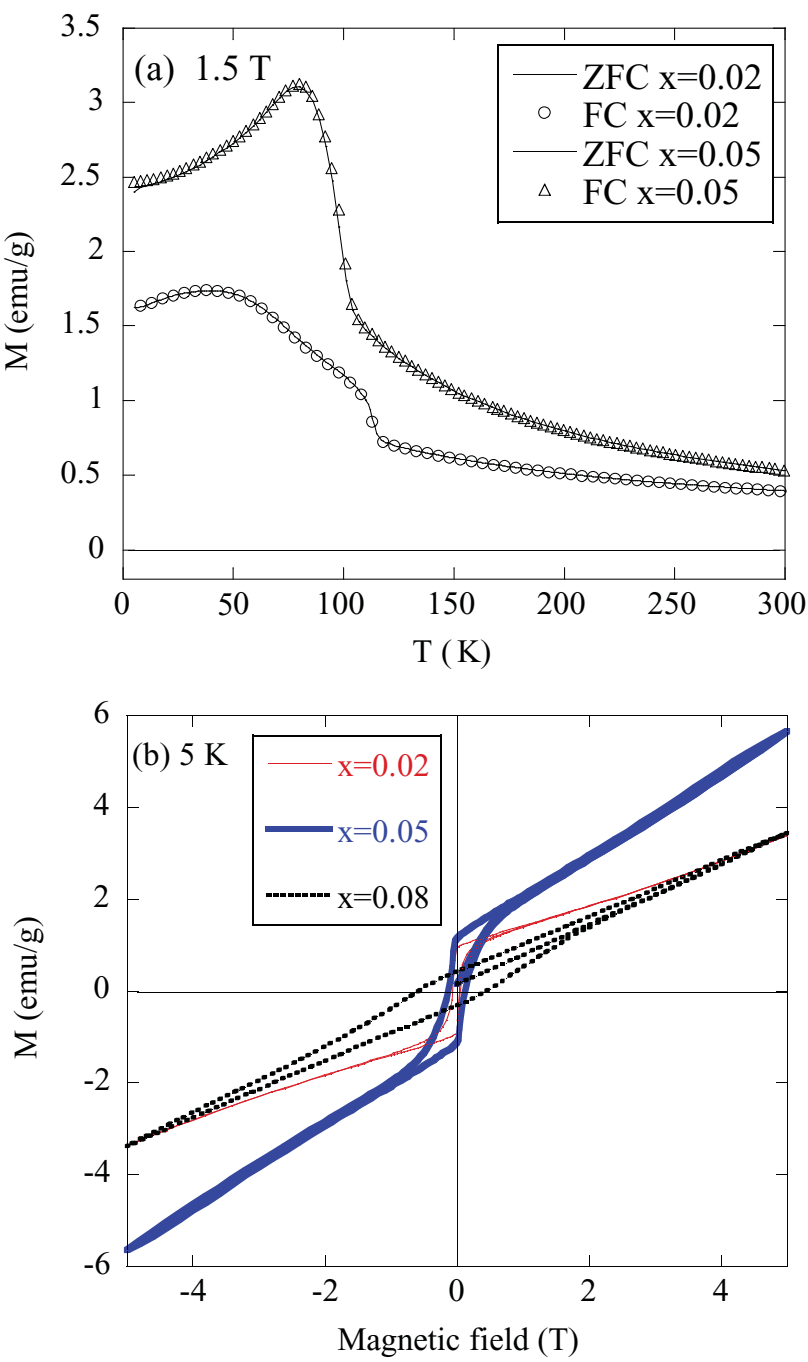

FIG. 7. (Color online) (a) Temperature dependence of ZFC and FC magnetization curves of the $\mathrm{CaMn}_{1-x} \mathrm{Sb}_{x} \mathrm{O}_{3}(x=0.02$ and 0.05) samples under an applied field of $1.5 \mathrm{~T}$. (b) Magnetization curves $M$ vs $H$ of $x=0.02,0.05$, and 0.08 at $5 \mathrm{~K}$.

configuration of $\mathrm{Mn}, \mu\left(\mathrm{Mn}^{4+}\right)=3.87 \mu_{B}$ and $\mu\left(\mathrm{Mn}^{3+}\right)=$ $4.90 \mu_{B}$. In the paramagnetic regime, we obtain $\Theta$ from the negative value of $-119 \mathrm{~K}$ at $x=0.02$ to the positive one of $28 \mathrm{~K}$ at $x=0.08$, which is indicative of a variation of the magnetic interaction from antiferromagnetic to ferromagnetic character. Furthermore, the effective magnetic moment shows a slight increase from $3.51 \mu_{B}$ at $x=0.02$ up to $3.70 \mu_{B}$ at $x=0.08$. We suppose the valence fluctuation of the $\mathrm{Sb}$ ion, to account for discrepancies in the effective moment between the experimental and calculated values as listed in Table III. In a recent study of $\mathrm{CaMn}_{1-x} \mathrm{Ru}_{x} \mathrm{O}_{3},{ }^{13} \mathrm{X}$-ray absorption measurements reveal the presence of the mixed valence states of the $\mathrm{Ru}$ ion. In the intermediate samples $(0.1 \leqslant x \leqslant 0.9)$, it has been reported that the valence states of $\mathrm{Ru}^{5+}$ and $\mathrm{Ru}^{4+}$ coexist with the mixed valence of $\mathrm{Mn}$ ion. However, $\mathrm{x}$-ray photoelectron spectroscopy analysis of $\mathrm{La}_{0.9} \mathrm{Sb}_{0.1} \mathrm{MnO}_{3}{ }^{44}$ indicates that the valence state of $\mathrm{Sb}$ ion is +5 . Thus, we believe that $\mathrm{Sb}^{5+}$ exists predominantly in the low-doping region of $\mathrm{Sb}$. These differences seem to point to the existence of ferromagnetic fluctuation or the formation of 


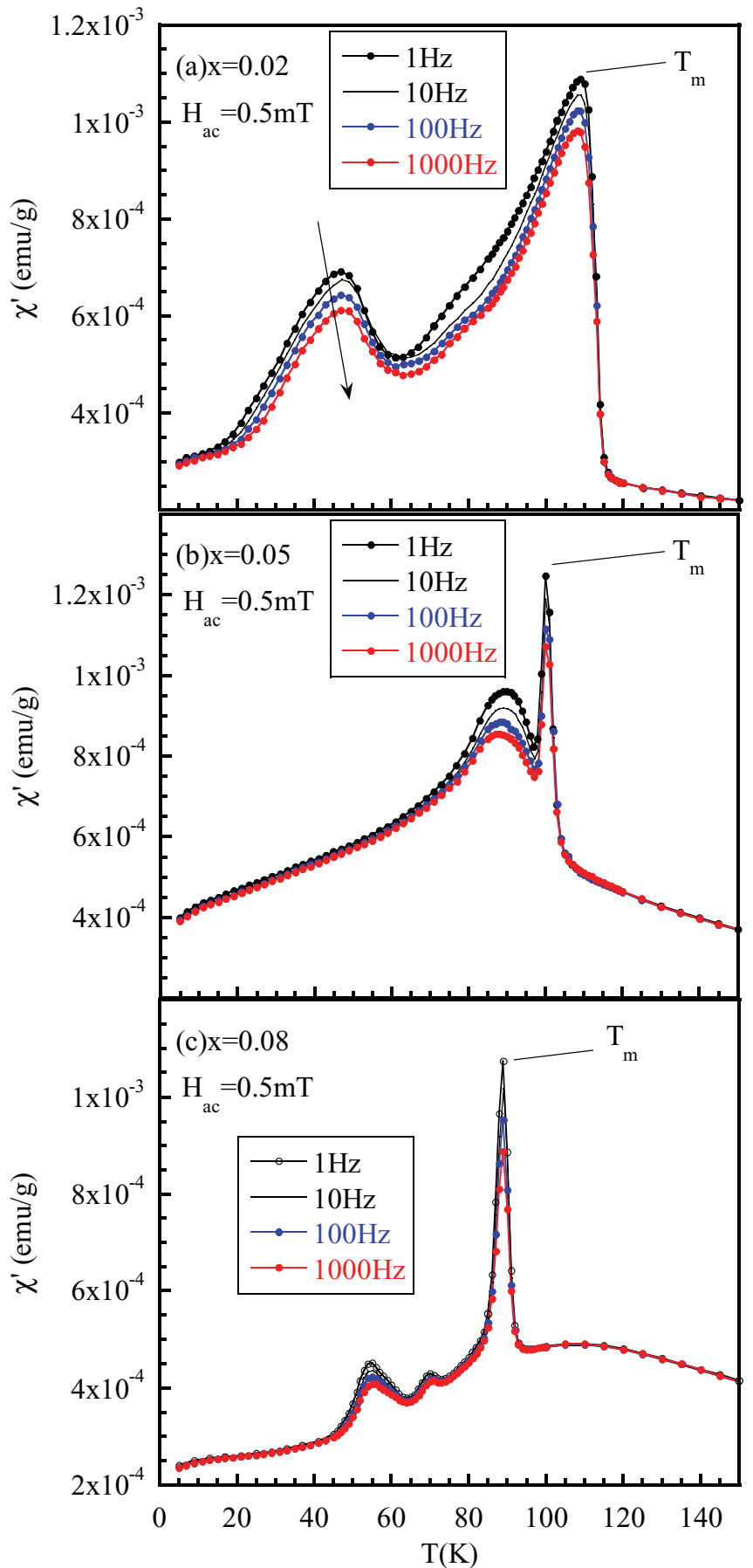

FIG. 8. (Color online) The real part $\chi^{\prime}$ of ac magnetic susceptibility of $\mathrm{CaMn}_{1-x} \mathrm{Sb}_{x} \mathrm{O}_{3}$ collected at zero dc magnetic field with frequency ranging from $1 \mathrm{~Hz}$ to $1000 \mathrm{~Hz}$. (a) $x=0.02$, (b) $x=0.05$, and (c) $x=0.08$. The amplitude of the ac magnetic field $H_{\mathrm{ac}}$ was set to be $0.5 \mathrm{mT}$. The arrows point to the direction of increasing frequencies.

FM small clusters, in the paramagnetic phase of the Sb doped sample above the magnetic transition temperature.

In a relatively high field of $1.5 \mathrm{~T}$, the temperature variation of the magnetization for $x=0.02$ and 0.05 shows no magnetic hysteresis between the ZFC and FC curves as displayed in Fig. 7(a). The $M(H)$ data with a small hysteresis do not saturate even at $5 \mathrm{~T}$ and rise linearly with increasing $H$ as shown

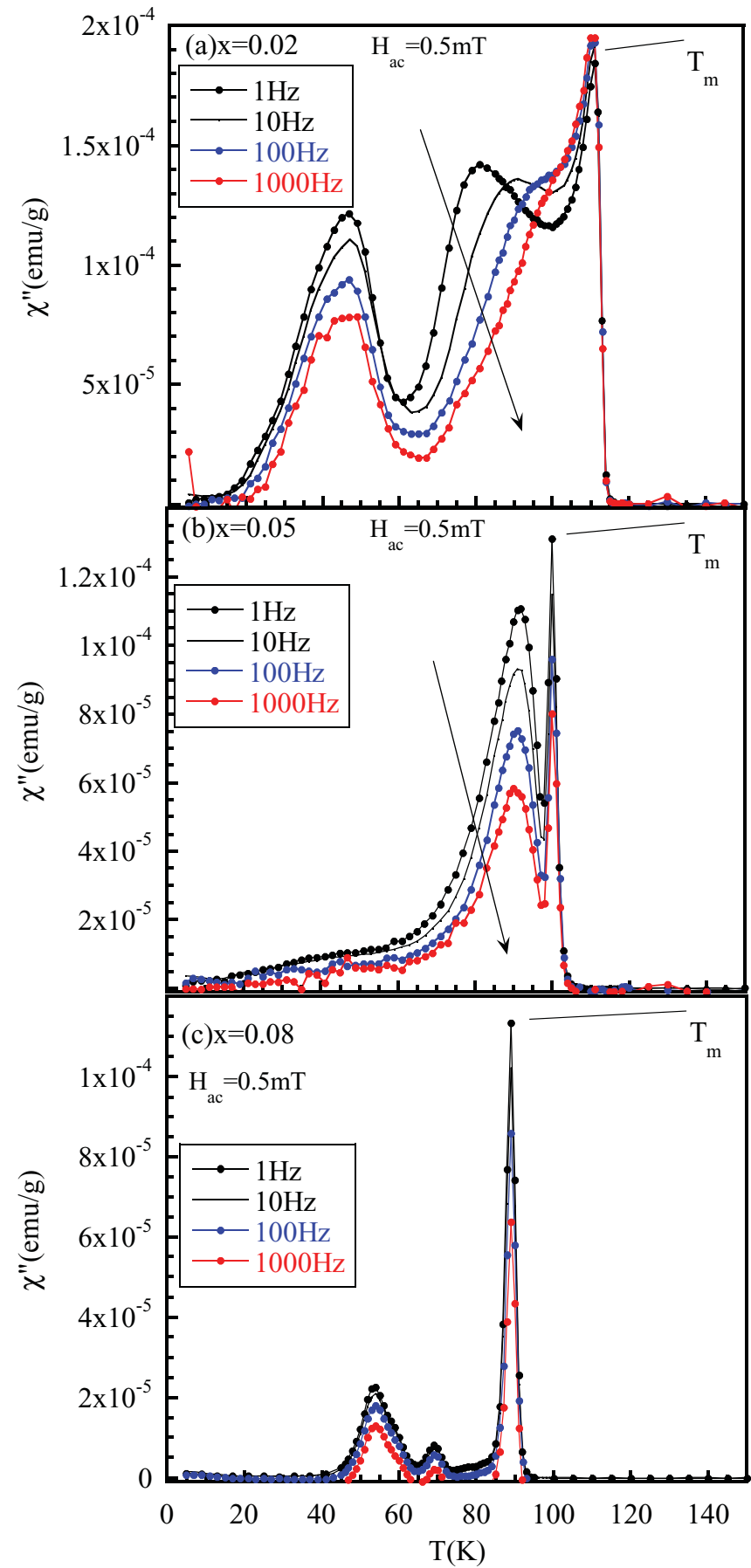

FIG. 9. (Color online) The imaginary part $\chi^{\prime \prime}$ of the ac magnetic susceptibility of $\mathrm{CaMn}_{1-x} \mathrm{Sb}_{x} \mathrm{O}_{3}$ as a function of frequency varying from $1 \mathrm{~Hz}$ to $1000 \mathrm{~Hz}$ at $H_{\mathrm{dc}}=0 \mathrm{~T}\left(H_{\mathrm{ac}}=0.5 \mathrm{mT}\right)$. (a) $x=0.02$, (b) $x=0.05$, and (c) $x=0.08$.

in Fig. 7(b), indicating the AFM matrix with the small FM region. We understand from both Figs. 2(c) and 7(b) that the larger magnetization at high fields corresponds to the stronger magnetoresistance effect. A linear extrapolation of the $M(H)$ curve at $5 \mathrm{~K}$ to $H=0$ gives a spontaneous magnetization $M_{s}$ from $0.97 \mathrm{emu} / \mathrm{g}$ at $x=0.02$ through $1.2 \mathrm{emu} / \mathrm{g}$ at $x=0.05$ down $0.42 \mathrm{emu} / \mathrm{g}$ at $x=0.08$, revealing the existence of small residual magnetization for all samples studied. 


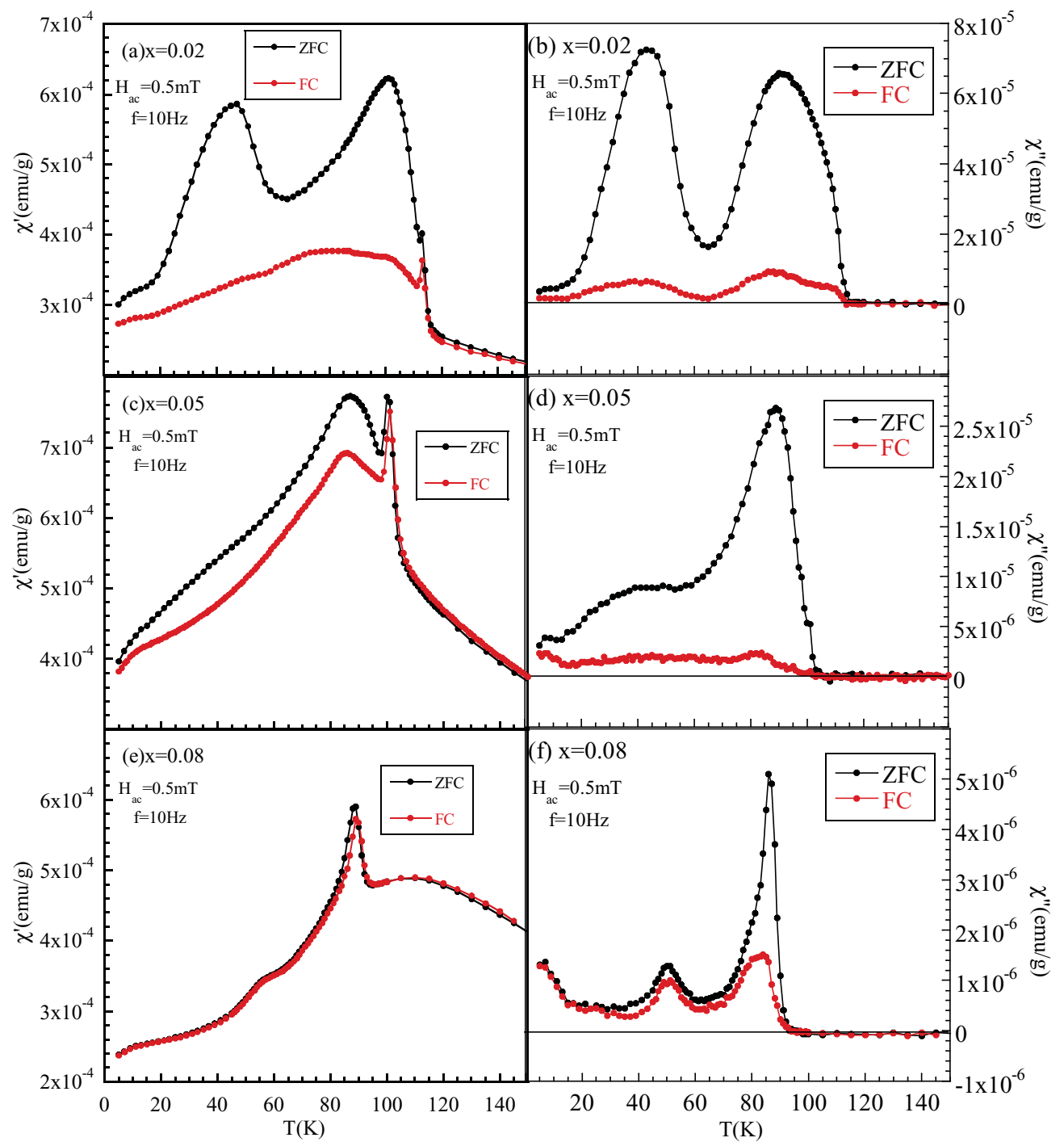

FIG. 10. (Color online) The real and imaginary parts of ac magnetic susceptibility of $\mathrm{CaMn}_{1-x} \mathrm{Sb}_{x} \mathrm{O}_{3}$ measured at $10 \mathrm{~Hz}$ under a superimposed dc field $\left(H_{\mathrm{dc}}=10 \mathrm{mT}\right.$ ). For $x=0.02$, (a) $\chi^{\prime}$ and (b) $\chi^{\prime \prime}$. For $x=0.05$, (c) $\chi^{\prime}$ and (d) $\chi^{\prime \prime}$. For $x=0.08$, (e) $\chi^{\prime}$ and (f) $\chi^{\prime \prime}$. The ac magnetization data are recorded as a function of temperature under ZFC and FC conditions.

\section{Frequency and dc magnetic field dependences of ac magnetic susceptibility}

Furthermore, we carry out the ac magnetic susceptibility measurements for $x=0.02,0.05$, and 0.08 samples, in order to examine the dynamic effect linked to magnetically frustrated properties (Figs. 8 and 9). The real and imaginary parts of the ac susceptibility, $\chi^{\prime}$ and $\chi^{\prime \prime}$, are registered at zero dc magnetic field with frequency $f$ varying from $1 \mathrm{~Hz}$ to $1000 \mathrm{~Hz}$. For $x=0.02$, a sharp transition is noticed around $T_{m}=110 \mathrm{~K}$, which agrees well with the dc magnetic measurements. A maximum of the $\chi^{\prime}$ curves shows no shift in temperature with increasing $f$ but a second peak at lower temperature exhibits a small shift toward high temperatures, indicating the presence of a magnetic frustration. The magnitude of the $\chi^{\prime}(T)$ curves is suppressed with frequency over a wide range of temperatures. In addition to the two peaks observed in $\chi^{\prime}$, a third peak in $\chi^{\prime \prime}$ appears at low frequencies, its peak then shifts toward higher temperatures with increasing $f$, and finally it is merged in the maximum of the peak at high frequencies. The new peak around $80 \mathrm{~K}$ at $1 \mathrm{~Hz}$ strongly depends on frequency. The finite imaginary component representing magnetic energy dissipation is closely related to the existence of magnetic hysteresis between the $\mathrm{ZFC}$ and $\mathrm{FC}$ dc magnetization curves. For $x=0.05$, the maximum of the peak is located at $T_{m}=$ $100 \mathrm{~K}$, while the second one observed at a higher temperature of $90 \mathrm{~K}$ is accompanied by a slight frequency shift toward low temperatures. It seems that the frequency shift of the second peak in the $\chi^{\prime}$ and $\chi^{\prime \prime}$ curves for both $x=0.02$ and 0.05 is indicative of the signature of a spin-glass-like magnetic property. However, a substantial decrease of the peak in $\chi^{\prime \prime}$ with increasing frequency is qualitatively in contrast to the behavior of conventional spin-glass system, ${ }^{30}$ as previously reported in phase separated $\operatorname{Pr}_{0.7} \mathrm{Ca}_{0.3} \mathrm{MnO}_{3} .{ }^{31}$ The dynamical effect of $\chi^{\prime}$ has some relationship with the formation of the magnetically frustrated clusters such as FM clusters embedded in canted AFM matrix. On the other hand, in the case of $x=0.08$, the second peak in the $\chi^{\prime}$ and $\chi^{\prime \prime}$ curves is located near $55 \mathrm{~K}$ and it shows a weak frequency dependence as 


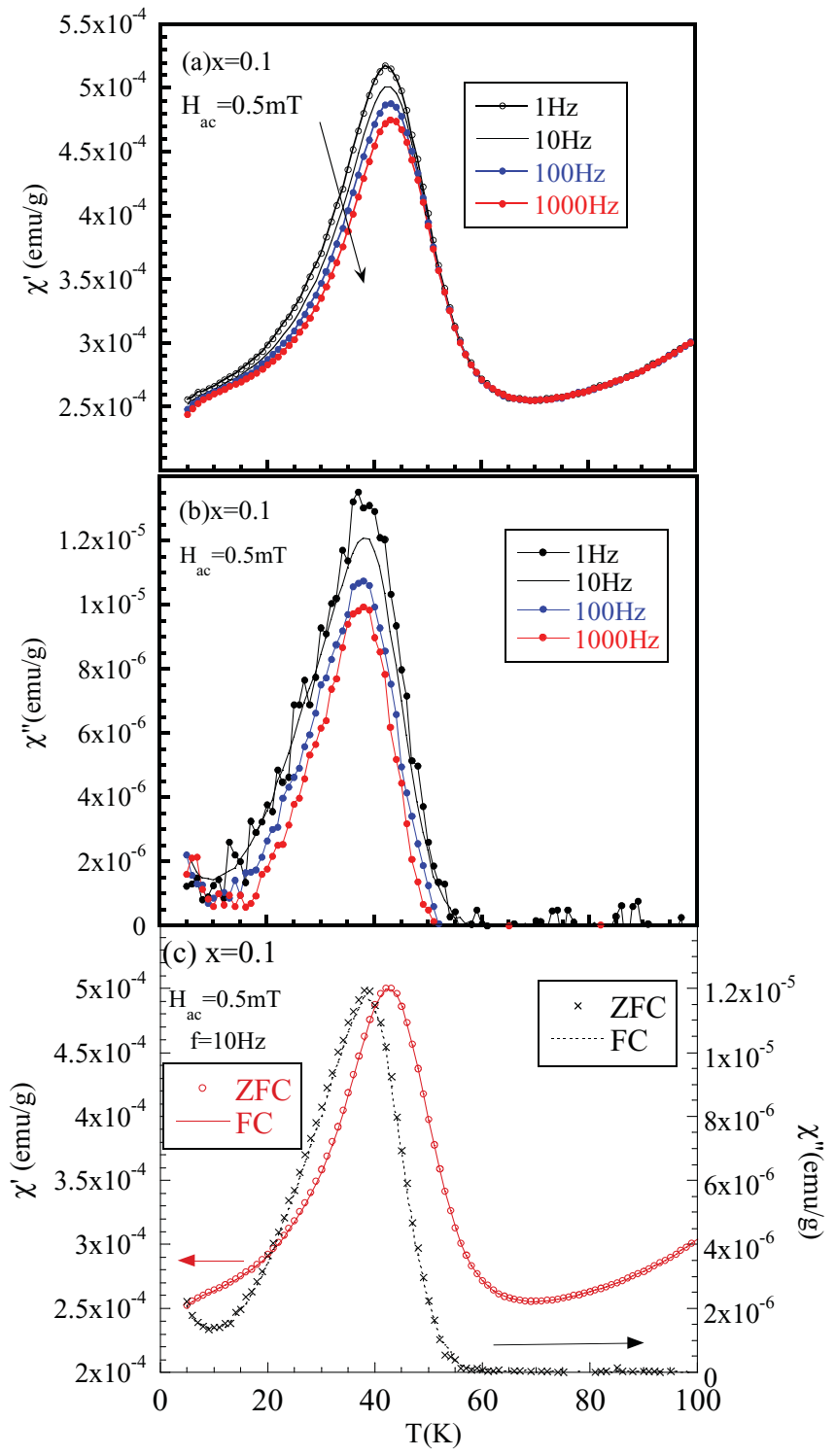

FIG. 11. (Color online) The real and imaginary parts of ac magnetic susceptibility of $\mathrm{CaMn}_{0.9} \mathrm{Sb}_{0.1} \mathrm{O}_{3}$ a function of frequency varying from $1 \mathrm{~Hz}$ to $1000 \mathrm{~Hz}$ at $H_{\mathrm{dc}}=0 \mathrm{~T}\left(H_{\mathrm{ac}}=0.5 \mathrm{mT}\right)$. For $x=0.1$, (a) $\chi^{\prime}$ and (b) $\chi^{\prime \prime}$. For comparison, the ac magnetization data recorded as a function of temperature under ZFC and FC conditions are given in (c) $\left(H_{\mathrm{dc}}=10 \mathrm{mT}\right)$.

shown in Figs. 8 and 9. We notice that the second peaks of $x=0.02$ and 0.05 are located around $47 \mathrm{~K}$ and $90 \mathrm{~K}$, respectively, corresponding to the maximum of the $\mathrm{ZFC} \mathrm{dc}$ magnetization. However, the second peak of $x=0.08$ has no corresponding maximum in the ZFC dc curve [Fig. 5(c)]. We note that the ac susceptibility of the parent $\mathrm{CaMnO}_{3}$ exhibits no magnetic peak except for a peak associated with long-range magnetic ordering. ${ }^{35}$ Moreover, for the B-site substituted $\mathrm{CaMn}_{1-x} \mathrm{Mo}_{x} \mathrm{O}_{3}(x=0.04)$, the imaginary part $\chi^{\prime \prime}$ shows only a sharp peak at $105 \mathrm{~K}$ around the magnetic transition temperature and no signal at low temperatures. ${ }^{18}$ For the low-doped $\mathrm{CaMn}_{1-x} \mathrm{Ru}_{x} \mathrm{O}_{3}(x=0.06)$, both $\chi^{\prime}$ and $\chi^{\prime \prime}$ exhibit neither frequency dependence nor an enhanced peak below $T_{m} .{ }^{10}$ This finding predicts that the low-temperature ground state of the light $\mathrm{Ru}$ substituted $\mathrm{CaMnO}_{3}$ is better explained by the complex canted magnetic structure than by the phase separated model. For A-site substituted $\mathrm{Ca}_{0.9} \mathrm{La}_{0.1} \mathrm{MnO}_{3}$, a much larger hysteresis in the dc magnetization between ZFC and FC curves, and the frequency effect on the ac susceptibility demonstrate that its low-temperature state is considered as a cluster glass one without long-range ferromagnetism. ${ }^{35}$

Now, under zero-field-cooled and field-cooled conditions, we display in Fig. 10 the ac susceptibility measurements of $x=0.02,0.05$, and 0.08 with a superimposed dc field $\left(H_{\mathrm{dc}}=\right.$ $10 \mathrm{mT}$ ). First of all, the sharp peaks in both $\chi^{\prime}$ and $\chi^{\prime \prime}$ around $T_{m}$ in the absence of dc field are strongly suppressed under the presence of the dc field but the second peaks of $x=0.02$ and 0.05 still remain stable. The collapse of the steep peak at zero dc field is caused by the application of low dc field since the applied low field strongly suppresses the magnetic fluctuation associated with the magnetic transition. ${ }^{45} \mathrm{Next}$, a magnetic divergence in the ac magnetization between the $\mathrm{ZFC}$ and FC curves is visible at $x=0.02$, but upon increasing the Sb content, both the ZFC and FC ac curves at $x=0.08$ become reversible and exhibit no clear differences except for the high temperature region near $T_{m}$. Finally, for the $x=0.08$ sample the maximum of the peak in FC $\chi^{\prime \prime}$ of Fig. 10(a) is observed around $85 \mathrm{~K}$, at the temperature where the FC dc curve reaches a maximum as shown in Fig. 5(e). If we assume that the peak in FC $\chi^{\prime \prime}$ is related to the formation of magnetically frustrated clusters associated with a phase-separated state, we cannot identify the second peak with corresponding signature in the dc magnetization curve. However, the anomalous peak is located near the characteristic temperature pointing to the dc magnetization reversal. Accordingly, we suppose that the existence of the anomalous peak in $\chi^{\prime \prime}$ of $x=0.08$ has some relationship with the negative magnetization phenomena observed here. For comparison, the ac susceptibility data of the $x=0.1$ sample are presented in Fig. 11. We notice the frequency dependence of the ac peak in both $\chi^{\prime}$ and $\chi^{\prime \prime}$ around $40 \mathrm{~K}$ corresponding to the ZFC dc peak in the inset of Fig. 5(e), indicating the strong evidence for a magnetically frustrated state.

\section{SUMMARY}

We have demonstrated the effect of hydrostatic pressure on magnetic and transport properties of the electron-doped manganite $\mathrm{CaMn}_{1-x} \mathrm{Sb}_{x} \mathrm{O}_{3}$. In addition, thermal transport properties (Seebeck coefficient and thermal conductivity) of the $\mathrm{CaMn}_{1-x} \mathrm{Sb}_{x} \mathrm{O}_{3}$ system have been examined as a function of $T$. Furthermore, the ac magnetic susceptibility measurements for $x=0.02,0.05$, and 0.08 samples have been performed, in order to examine a close relation between the dynamic effect linked to magnetically frustrated properties and the static de magnetization.

The substitution of the $\mathrm{Sb}^{5+}$ ion for $\mathrm{Mn}^{4+}$ site of the parent matrix causes one-electron doping with the chemical formula $\mathrm{CaMn}_{1-2 x}^{4+} \mathrm{Mn}_{x}^{3+} \mathrm{Sb}_{x}^{5+} \mathrm{O}_{3}$ accompanied by a monotonous increase in unit-cell volume as a function of $x$. Upon increasing the doping level of $\mathrm{Sb}$, the magnitudes of both electrical resistivity and the Seebeck coefficient are suppressed at high temperatures, indicating the electron doping. The CM model applied to the random hopping system gives a better fit to the Seebeck coefficient at higher temperatures. The light doping 
of $\mathrm{Sb}^{5+}$ strongly suppresses the high-thermal conductivity of the parent sample through the local lattice distortions.

The anomalously diamagnetic behaviors at $x=0.05$ and 0.08 are clearly observed in the field-cooled dc magnetization. The magnetization curves of $x=0.05$ and 0.08 under an applied pressure of $0.8 \mathrm{GPa}$ exhibit similar behaviors to those of the $x=0.02$ and 0.05 samples without pressure, respectively. We expect that these findings are close to some change of the local easy axis of magnetization due to the local lattice distortion induced by the $\mathrm{Sb}$ doping, in comparison to the case of the A-site substitution. A notable difference in the magnetization curves between the $x=0.1$ sample and other ones is attributed to the weakened magnetic interaction working among the $\mathrm{Mn}$ ions around the $\mathrm{Sb}$ ion.
The dynamical effect of the ac magnetic susceptibility measurement has some relationship with the formation of the magnetically frustrated clusters such as FM clusters embedded in canted AFM matrix. In particular, we suppose that the anomalous peak in FC $\chi^{\prime \prime}$ of $x=0.08$ is related to the negative magnetization observed here, suggesting the metastable and nonequilibrium nature of the above effect.

\section{ACKNOWLEDGMENTS}

This work was partially supported by a Grant-in-Aid for Scientific Research from Japan Society of the Promotion of Science. *matsukawa@iwate-u.ac.jp

†Present address: DOWA Electronics Materials Co., Ltd., Okayama 702-8045, Japan

${ }^{1}$ Colossal Magnetoresistive Oxides, edited by Y. Tokura (Gordon and Breach, New York, 2000).

${ }^{2}$ C. Zener, Phys. Rev. 82, 403 (1951); P. G. deGennes, ibid. 118, 141 (1960).

${ }^{3}$ E. Dagotto, Nanoscale Phase Separation and Colossal Magnetoresistance (Springer, Berlin, 2003).

${ }^{4}$ A. J. Millis, P. B. Littlewood, and B. I. Shraiman, Phys. Rev. Lett. 74, 5144 (1995); A. J. Millis, B. I. Shraiman, and R. Mueller, ibid. 77, 175 (1996).

${ }^{5}$ J. B. MacChesney, H. J. Williams, J. F. Potter, and R. C. Sherwood, Phys. Rev. 164, 779 (1967).

${ }^{6}$ H. Chiba, M. Kikuchi, K. Kasuba, Y. Muraoka, and Y. Syono, Solid State Commun. 99, 499 (1996).

${ }^{7}$ E. Granado, C. D. Ling, J. J. Neumeier, J. W. Lynn, and D. N. Argyriou, Phys. Rev. B 68, 134440 (2003).

${ }^{8}$ B. Raveau, Y. M. Zhao, C. Martin, M. Hervieu, and A. Maignan, J. Solid State Chem. 149, 203 (2000).

${ }^{9}$ L. Pi. S. Hebert, C. Martin, A. Maignan, and B. Raveau, Phys. Rev. B 67, 024430 (2003).

${ }^{10}$ A. I. Shames, E. Rozenberg, C. Martin, A. Maignan, B. Raveau, G. Andre, and G. Gorodetsky, Phys. Rev. B 70, 134433 (2004).

${ }^{11}$ Y. Guo, W. Li, S. Roy, and N. Ali, Chem. Mater. 17, 2735 (2005).

${ }^{12}$ R. Ang, Y. P. Sun, Y. Q. Ma, B. C. Zhao, X. B. Zhu, and W. H. Song, J. Appl. Phys. 100, 063902 (2006).

${ }^{13}$ Q. Zhou, B. J. Kennedy, Z. Zhang, L. Y. Jang, and J. B. Aitken, Chem. Mater. 21, 4203 (2009).

${ }^{14}$ C. Martin, A. Maignan, M. Hervieu, B. Raveau, J. Hejtamanek, Phys. Rev. B 63, 100406 (2001).

${ }^{15}$ A. Maignan, C. Martin, C. Autret, M. Hervieu, B. Raveau, and J. Hejtmanek, J. Mater. Chem. 12, 1806 (2002).

${ }^{16}$ M. Miclau, J. Hejtmanek, R. Retoux, K. Knizek, Z. Zirak, R. Fresard, A. Maignan, S. Hebert, M. Hervieu, and C. Martin, Chem. Mater. 19, 4243 (2007).

${ }^{17}$ E. Rozenberg, M. Auslender, A. I. Shames, C. Martin, and S. Hebert, J. Appl. Phys. 103, 07F720 (2008).

${ }^{18}$ C. Martin, M. Miclau, S. Hebert, M. Giot, A. Maignan, G. Andre, and F. Bouree-Vigneron, J. Magn. Magn. Mater. 321, 3938 (2009).

${ }^{19}$ H. Kuwahara, Y. Moritomo, Y. Tomioka, A. Asamitsu, M. Kasai, R. Kumai, and Y. Tokura, Phys. Rev. B 56, 9386 (1997).
${ }^{20}$ V. Markovich, I. Fita, R. Puzniak, E. Rozenberg, C. Martin, A. Wisniewski, A. Maignan, B. Raveau, Y. Yuzhelevskii and G. Gorodetsky, Phys. Rev. B 70, 024403 (2004); V. Markovich, I. Fita, R. Puzniak, E. Rozenberg, C. Martin, A. Wisniewski, Y. Yuzhelevskii, and G. Gorodetsky, ibid. 71, 134427 (2005).

${ }^{21}$ Y. Murano, M. Matsukawa, S. Kobayashi, S. Nimori, and R. Suryanarayanan, J. Phys. Conf. Series 200, 012114 (2010).

${ }^{22}$ F. Bartolome, J. Herrero-Albillos, L. M. Garcia, J. Bartolome, N. Jaouen, and A. Rogalev, J. Appl. Phys. 97, 10A503 (2005).

${ }^{23}$ I. O. Troyanchuk, V. A. Khomchenko, S. N. Pastushonok. O. A. Novitsky, V. I. Pavlov, and H. Szymczak, J. Magn. Magn. Mater. 303, 111 (2006).

${ }^{24}$ J. Hemberger, S. Lobina, H. A. Krug von Nidda, N. Tristan, V. Yu. Ivanov, A. A. Mukhin, A. M. Balbashov, and A. Loidl, Phys. Rev. B 70, 024414 (2004).

${ }^{25}$ I. O. Troyanchuk, V. A. Khomchenko, G. M. Chobot, A. I. Kurbakov, A. N. Vasilev, V. V. Eremenko, V. A. Sirenko, M. Yu. Shvedun, H. Szymczak, and R. Szymczak, J. Phys. Condens. Matter 15, 8865 (2003).

${ }^{26}$ O. Peña, M. Bahout, K. Ghanimi, P. Duran, D. Gutierrez, and C. Moure, J. Mater. Chem. 12, 2480 (2002); O. Pena, C. Moure, P. Barahona, M. Baibich, and G. Martinez, Physica B 384, 57 (2006).

${ }^{27}$ C. A. Nordman, V. S. Achutharaman, V. A. Vasko, P. A. Kraus, A. R. Ruosi, A. M. Kadin, and A. M. Goldman, Phys. Rev. B 54, 9023 (1996).

${ }^{28}$ M. Mouallem-Bahout, O. Pena, D. Gutierrez, P. Duran, and C. Moure, Solid State Commun. 122, 561 (2002).

${ }^{29}$ V. Markovich, I. Fita, A. Wisniewski, R. Puzniak, D. Mogilyansky, L. Titelman, L. Vradman, M. Herskowitz, and G. Gorodetsky, Phys. Rev. B 77, 014423 (2008)

${ }^{30}$ C. A. M. Mulder, A. J. van Duyneveldt, and J. A. Mydosh, Phys. Rev. B 23, 1384 (1981).

${ }^{31}$ I. G. Deac, J. F. Mitchell, and P. Schiffer, Phys. Rev. B 63, 172408 (2001).

${ }^{32}$ V. Poltavets, K. Vidyasagar, and M. Jansen, J. Solid State Chem. 177, 1285 (2004).

${ }^{33}$ In our previous work, ${ }^{21}$ we presented the low-field magnetization measurements by using the SQUID magnetometer. Recently, we noticed that the remanent magnetic field in the superconducting magnet affects the temperature variation of the magnetization in the zero-field-cooled runs. In fact, we checked it by using the SQUID 
magnetometer with the function of magnet reset mode, to reduce the remanent field below about $2 \mathrm{G}$. Separately, we tried to perform the magnetization measurements with the SQUID by using the fluxgate device for further reduction of the remanent value below $1 \mathrm{mG}$. The former result was in good agreement with the latter. As a result, we expect that the apparent "ZFC" scan in the previous measurements is a field-cooled one under the negative remanent field in the case of switching off the magnet reset.

${ }^{34}$ M. Jaime, M. B. Salamon, M. Rubinstein, R. E. Treece, J. S. Horwitz, and D. B. Chrisey, Phys. Rev. B 54, 11914 (1996).

${ }^{35}$ A. Maignan, C. Martin, F. Damay, B. Raveau, and J. Hejtmanek, Phys. Rev. B 58, 2758 (1998).

${ }^{36}$ Z. Zeng, M. Greenblatt, and M. Croft, Phys. Rev. B 59, 8784 (1999).

${ }^{37}$ Seebeck coefficient $S$ is a sensitive probe to examine the electronic state of disordered crystals or highly inhomogeneous materials. $S$ is less affected by grain boundaries of polycrystalline samples than the electrical conductivity measurement since the thermal current applied to the sample is hard to be disturbed by the grain boundaries in comparison to the electric current.

${ }^{38}$ B. Fisher, L. Patlagan, G. M. Reisner, and A. Knizhnik, Phys. Rev. B 61, 470 (2000).

${ }^{39}$ I. Maekawa, F. Takagi, Y. Sakai, and N. Tsuda, J. Phys. Soc. Jpn. 56, 2119 (1987).

${ }^{40}$ M. Culter and N. F. Mott, Phys. Rev. 181, 1336 (1969).

${ }^{41}$ J. Hejtmanek, Z. Jirak, M. Marysko, C. Martin, A. Maignan, M. Hervieu, and B. Raveau, Phys. Rev. B 60, 14057 (1999).

${ }^{42}$ J. L. Cohn and J. J. Neumeier, Phys. Rev. B 66, 100404(R) (2002).

${ }^{43}$ M. Matsukawa, M. Narita, T. Nishimura, M. Yoshizawa, M. Apostu, R. Suryanarayanan, A. Revcolevschi, K. Itoh, and N. Kobayashi, Phys. Rev. B 67, 104433 (2003).

${ }^{44}$ P. Duan, S. Y. Dai, G. T. Tan, H. B. Lu, Y. L. Zhou, B. L. Cheng, and Z. H. Chen, J. Appl. Phys. 95, 5666 (2004).

${ }^{45}$ S. Mukherjee, R. Ranganathan, P. S. Anilkumar, and P. A. Joy, Phys. Rev. B 54, 9267 (1996). 